\title{
Exenatide exerts a neuroprotective effect against diabetic cognitive impairment in rats by inhibiting apoptosis: Role of the JNK/c-JUN signaling pathway
}

\author{
GENGYIN WANG $^{1 *}$, ZONGQUAN ZHAO $^{2 *}$, BO REN $^{3}$, WU YU $^{4}$, XUDONG ZHANG $^{1}$, \\ JIANG LIU ${ }^{1}$, LIPING WANG ${ }^{1}$, DAOWEN SI ${ }^{1}$ and MEILIU YANG ${ }^{5}$
}

\begin{abstract}
${ }^{1}$ Department of Human Anatomy, School of Basic Medical Sciences, North China University of Science and Technology, Tangshan, Hebei 063210; ${ }^{2}$ General Practice, Pingjiang Xincheng Community Health Service Center, Suzhou, Jiangsu 215101;

${ }^{3}$ Medical Experimental Center, Jitang College of North China University of Science and Technology, Tangshan, Hebei 063210; ${ }^{4}$ School Hospital, Hengshui University; ${ }^{5}$ Department of Life Sciences, Hengshui University, Hengshui, Hebei 053010, P.R. China
\end{abstract}

Received July 21, 2021; Accepted November 25, 2021

DOI: $10.3892 / \mathrm{mmr} .2022 .12627$

\begin{abstract}
Exenatide could reduce blood glucose and alleviate cognitive dysfunction induced by diabetes mellitus (DM). In the present study, a diabetic model was established in Sprague-Dawley rats to further explore the mechanism of exenatide on diabetes-induced cognitive impairment. Notably, the model rats performed poorly in the Morris water maze test and had more apoptotic neurons compared with the control rats. By contrast, exenatide attenuated cognitive impairment and inhibited neuronal apoptosis in the DM rat model. To explore the neuroprotective mechanisms of exenatide, western blotting was performed to detect the expression levels of markers of endoplasmic reticulum stress, including cytochrome $c$ (Cyt-c), Caspase-3, JNK and c-JUN, in hippocampal tissue. Reverse transcription-quantitative PCR was also performed to measure the mRNA expression levels of Cyt-c and Caspase-3. After 16 weeks of treatment, exenatide treatment downregulated Cyt-c, Caspase-3, phosphorylated (p)-JNK and p-c-JUN expression in the hippocampal tissue of diabetic rats. Moreover, Cyt-c, Caspase-3, JNK and JUN expression levels were detected following treatment with a specific inhibitor of JNK (SP600125). The results revealed that SP600125 had similar
\end{abstract}

Correspondence to: Dr Meiliu Yang, Department of Life Sciences, Hengshui University, 1088 Heping West Road, Hengshui, Hebei 053010, P.R. China

E-mail: yangmeiliu99@163.com

*Contributed equally

Abbreviations: DM, diabetes mellitus; ER, endoplasmic reticulum; ERS, ER stress; MCI, mild or moderate cognitive impairment; MWM, Morris water maze

Key words: exenatide, diabetes, cognitive impairment, JNK/c-JUN signaling pathway, apoptosis inhibitory effects on the JNK pathway and ERS-related protein expression (Cyt-t, Caspase-3, p-JNK and p-c-JUN). These results suggested that exenatide improved cognitive dysfunction in DM rats and that the underlying mechanism may be associated with inhibiting apoptosis by suppressing the activation of JNK/c-JUN.

\section{Introduction}

In recent years, diabetic encephalopathy has become a common but often overlooked chronic diabetic complication in clinical practice, and cognitive dysfunction is its main clinical characteristic $(1,2)$. According to epidemiological data, $60-70 \%$ of patients with DM have mild or moderate cognitive impairment (MCI), which significantly increases the risk of dementia and imposes a heavy burden on the families of patients and society $(3,4)$. Previous studies have shown that as the disease progresses, patients with diabetes gradually exhibit cognitive impairments, such as declining learning and memory ability, and spatial cognitive ability $(5,6)$. It has been previously reported that the incidence of cognitive impairment in patients with diabetes was twice that in a normal control group (7). Similar pathological changes have been observed in rats with type 2 diabetes and Alzheimer's disease (8).

At present, the pathogenesis of diabetic cognitive dysfunction remains unclear; however, it is considered to be related to numerous factors, such as abnormal glucose and lipid metabolism, oxidative stress, cerebrovascular disease and neuronal dysfunction $(9,10)$. The hippocampus is an important anatomical structure associated with learning and memory. Previous studies have confirmed that diabetic hyperglycemia may lead to the degeneration, dysfunction and even apoptosis of hippocampal cells, and is one of most the important factors of diabetic cognitive impairment $(11,12)$. The endoplasmic reticulum (ER) is involved in an apoptosis pathway that differs from the mitochondrial and death receptor pathways; the core of this pathway is ER stress (ERS). Notably, the role of ERS in 
the occurrence and development of neurological diseases has attracted wide attention $(13,14)$.

Exenatide, a synthetic derivative of exendin-4, is a novel glucose-lowering drug. Exenatide is a glucagon-like peptide similar to GLP-1 that can bind to and activate the GLP-1 receptor (GLP-1R), and exert biological effects through cyclic adenosine phosphate or other signal transduction pathways. Previous studies have shown that GLP-1 can reduce blood glucose in patients with type 2 diabetes, improve dysfunctional lipid metabolism, increase the sensitivity of target tissues and target organs to insulin, and reduce insulin resistance $(15,16)$. Moreover, the GLP-1R agonist exenatide has been reported to ameliorate myocardial fibrosis in diabetic rats $(17,18)$. It has been demonstrated that GLP-1 and GLP-1Rs are widely expressed throughout the central nervous system, suggesting that the GLP-1/GLP-1R pathway may be involved in regulation of central nervous system function $(17,18)$. The GLP-1/GLP-1 $\mathrm{R}$ pathway may also be involved in the role of central insulin and in the regulation of learning and memory functions (19). Furthermore, GLP-1 may exert neurotrophic and neuroprotective effects that are closely related to the plasticity and survival of neurons (20). GLP-1 and its analogs, such as exendin-4, can activate GLP-1Rs, upregulate the levels of cAMP in neurons, regulate the homeostasis of intracellular calcium ions, reduce the excitotoxicity of nerve cells, inhibit neuronal apoptosis, and promote the proliferation and differentiation of nerve cells (21). In addition, GLP-1 has been reported to inhibit the apoptosis of hippocampal nerve cells, as well as learning and memory dysfunction induced by microwave exposure in rats, and to protect nerve cells (22). Diabetic encephalopathy is a complication of diabetes and cognitive impairment is its main manifestation. In the present study, the effect of exenatide, a GLP-1 analog, on cognitive function in diabetic rats was examined and the possible mechanism was explored from an antiapoptotic point of view.

\section{Materials and methods}

Experimental animals and grouping. A total of 80 male Sprague-Dawley rats (age, 8 weeks; weight, 260-300 g) supplied by the North China University of Science and Technology (Tangshan, China) were used in laboratory experiments. Rats were housed in constant temperature $\left(22-25^{\circ} \mathrm{C}\right)$ and humidity $(50-60 \%)$ under a 12 -h light-dark cycle with free access to food and water. After 1 week of acclimation, the rat model was established, and access to food and water was unrestricted. All animal procedures complied with the Regulations of the Experimental Animal Administration (approval no. LAEC-NCST-20200002). The research was approved by the Animal Ethics Committee of North China University of Science and Technology (approval no. LAEC-NCST-20200002).

The experiments were divided into two stages. First, to investigate the possible protective mechanism of exenatide (cat. no. Pep03601; NJPeptide) in a diabetes mellitus (DM) model rat, 60 rats were divided into four groups ( $n=15$ rats/group): The control (CON) group, the DM group, the exenatide + control $(\mathrm{Ex}+\mathrm{CON})$ group and the exenatide $+\mathrm{DM}(\mathrm{Ex}+\mathrm{DM})$ group DM modeling was performed as described previously (23). The rats in the DM and Ex + DM groups received streptozotocin
(STZ; 30 mg/kg; cat. no. ab142155; Abcam) by intraperitoneal injection and were offered a high-sugar and high-fat diet (basic feed $59 \%$, lard $18 \%$, sucrose $20 \%$, egg yolk $3 \%$; Shanghai R\&S Biotechnology Co., Ltd.) 4 weeks before STZ injection. The $\mathrm{CON}$ and $\mathrm{Ex}+\mathrm{CON}$ groups were given the same amount of citric acid buffer (through i.p. injection) and fed routinely. The blood glucose of the rats was measured, and a blood glucose level $>16.67 \mathrm{mmol} / \mathrm{l}$ was considered to indicate successful modeling. After establishing the model, the Ex $+\mathrm{CON}$ and $\mathrm{Ex}+\mathrm{DM}$ group rats were treated with exenatide $(3 \mu \mathrm{g} / \mathrm{kg}$, b.i.d.), whereas the $\mathrm{CON}$ and DM group rats were treated with the same quantity of physiological saline (through i.p. injection). The second stage of experiments was conducted to determine whether the JNK/c-JUN pathway contributed to the protective effects of exenatide in DM using a specific inhibitor of JNK (SP600125; cat. no. ab120065; Abcam). The rats were divided into four groups ( $\mathrm{n}=5$ rats/group): The DM group, the SP600125 + DM (SP + DM) group (treated with SP600125, $10 \mathrm{mg} / \mathrm{kg} / \mathrm{d}$; through i.p. injection), the Ex + DM group (treated with exenatide, $3 \mu \mathrm{g} / \mathrm{kg}$, b.i.d.), and the $\mathrm{SP}+\mathrm{DM}+\mathrm{Ex}$ group (treated with SP600125, $10 \mathrm{mg} / \mathrm{kg} / \mathrm{d}$ and exenatide, $3 \mu \mathrm{g} / \mathrm{kg} / \mathrm{d}$ through i.p. injection). DM modeling was performed as aforementioned. After 16 weeks of continuous subcutaneous injection of exenatide, body weight, were measured. Blood was collected from the tail vein $(5 \mu \mathrm{l})$ to measure blood glucose with a Glucometer (Sannuo Biosensing Co., Ltd.) and blood samples $(5 \mathrm{ml})$ from the heart were taken to detect blood lipids and insulin levels.

Morris water maze (MWM) test. Water was added to a pool $(60 \mathrm{~cm}$ in height and $120 \mathrm{~cm}$ in diameter) to a depth of $30 \mathrm{~cm}$ and a platform was placed $1 \mathrm{~cm}$ below the surface of the water. The experiment lasted for 6 days following the 16 week treatment period. The first 5 days were considered the training period, and the test was performed on day 6 . During the training period, a quadrant was randomly selected as the entry point, the rats were gently placed into the water facing the pool wall, and the time from entering the water to climbing onto the platform (i.e., the escape latency) was recorded. If the platform was not found within $60 \mathrm{sec}$, the rat was led to the platform and placed on the platform for $30 \mathrm{sec}$ and the escape latency was recorded as $60 \mathrm{sec}$. On day 6 , the platform was removed, and time spent in the platform quadrant and swimming speed were recorded. The experimental animals, except for those used in histological experiments, were sacrificed by cervical dislocation after anesthesia (pentobarbital, $60 \mathrm{mg} / \mathrm{kg}$ ).

Hematoxylin and eosin $(H \& E)$ staining. After the behavioral experiment, 5 rats in each group were intraperitoneally injected with pentobarbital $(60 \mathrm{mg} / \mathrm{kg}$; cat. no. H31020502; Shanghai Xinya Pharmaceutical Co., Ltd.) for deep anesthesia. Cardiac perfusion with $4 \%$ paraformaldehyde ( $\mathrm{pH} 7.4$ ) was performed on rats. Subsequently, the brain was collected and transferred to $4 \%$ paraformaldehyde and kept overnight at $4{ }^{\circ} \mathrm{C}$. The brain tissue was washed with tap water, dehydrated with an ethanol gradient, vitrified with dimethylbenzene and immersed in wax. Brain tissues were sectioned $(5 \mu \mathrm{m})$ and used for H\&E staining at $37^{\circ} \mathrm{C}$. $5 \%$ eosin solution $(5 \%)$ was added for $10 \mathrm{~min}$, after which samples were rinsed with purified water and redyed for 
2 min. The sections were observed under a light microscope. Using an a light microscope, the H\&E-stained specimens in each field of view at x400 magnification were subjected to morphometric analysis. The number of intact nerve cells in each field of view in each part of the hippocampus was counted, and 10 fields of view were counted for each specimen. The number of nerve cells in different parts of the hippocampus is expressed as the number of cells per field.

Reverse transcription-quantitative PCR (RT-qPCR) detection. Trizol (cat. no. R0016; Beyotime Institute of Biotechnology) was used to extract total RNA from hippocampal tissues, and RT was performed at $50^{\circ} \mathrm{C}$ for $30 \mathrm{~min}$ (BeyoFast ${ }^{\mathrm{TM}}$ SYBR-Green One-Step qRT-PCR Kit; cat. no. D7268M; Beyotime Institute of Biotechnology). According to the instructions of the qPCR kit (cat. no. D7268M; Beyotime Institute of Biotechnology), a $20-\mu 1$ reaction system was prepared. The primer sequences were designed with the help of Primer 5.0 software (Primer Premier) as follows: Caspase-3, forward, 5'-CCGAAACTC TTCATCATTCAGGC-3', reverse, 5'-GTTCCACTGTCTGTC TCAATACCG-3'; cytochrome $c$ (Cyt-c), forward, 5'-AAA AGGAGGCAAGCATAAGACTG-3', reverse, 5'-CTTGTT GGCATCTGTGTAAGAGAATC-3'; and $\beta$-actin, forward, 5'-CAACCGTGA AAAGATGACCCAGAT-3', reverse, 5'-CAACCGTGAAAAGATGACCCAGAT-3'. The reaction conditions were as follows: Pre-denaturation at $95^{\circ} \mathrm{C}$ for $4 \mathrm{~min}$; followed by 40 cycles of denaturation at $95^{\circ} \mathrm{C}$ for $30 \mathrm{sec}$ and then annealing/extension at $65^{\circ} \mathrm{C}$ for $30 \mathrm{sec}$. The fluorescence signal was detected at the end of each annealing cycle, and the critical point of detection was set as the cycle quantification value. Dissolution curve analysis was subsequently performed under the following conditions: $95^{\circ} \mathrm{C}$ for $15 \mathrm{sec}$; $60^{\circ} \mathrm{C}$ for $20 \mathrm{sec} ; 95^{\circ} \mathrm{C}$ for $15 \mathrm{sec}$. The $2^{-\Delta \Delta \mathrm{Cq}}$ method was used to calculate the relative changes of gene expression (24).

TUNEL staining. The level of apoptosis in each group was assessed using an In Situ Cell Death Detection Kit (cat. no. Roche-11684817910; Merck), according to the manufacturer's instructions. A total of 10 high magnification fields (magnification, x400) were randomly counted for each section, which were prepared as previously outlined, using a light microscope, and the apoptotic index was calculated by dividing the number of TUNEL-positive cells by the total number of cells counted. Each sample was tested three times and the average was obtained.

Detection of Caspase-3 activity. The isolated brain tissue of rats were placed on an ice plate and was homogenized in an ice bath. The homogenate was transferred to a 1.5-ml centrifuge tube and was incubated in an ice bath for $5 \mathrm{~min}$. Subsequently, the homogenate was centrifuged at $13,000 \times \mathrm{g}$ (RCF) for 10-15 min at $4^{\circ} \mathrm{C}$, and the supernatant was collected for later use. Caspase- 3 activity was tested using the Caspase- 3 kit (cat. no. C1116; Beyotime Institute of Biotechnology). Notably, Caspase-3 can catalyze production of yellow pNA from the substrate Ac-DEVD-pNA and yellow pNA has a strong absorption peak near the wavelength of $405 \mathrm{~nm}$; therefore, Caspase-3 activity can be detected by measuring the absorbance [optical density (OD)]. The calculation formula of Caspase-3 activity was as follows: Caspase -3 activity $=\{[(\mathrm{OD}$ sample-OD
blank)/OD blank] $\mathrm{x} 1,000\} /($ sample protein concentration $\mathrm{x}$ sampling liquid).

Western blotting. Hippocampal tissue was sliced into fragments and lysed with lysate to obtain total protein (cat. no. P0013E-1; Beyotime Institute of Biotechnology) and the protein concentration was analyzed using the Bradford method. Protein $(50 \mu \mathrm{g})$ was loaded and separated using a precast $12 \%$ SDS-PAGE gels. Subsequently, the PVDF membrane was blocked in 5\% skim milk powder solution at $37^{\circ} \mathrm{C}$ for 2 h. Caspase-3 (1:1,000; cat. no. ab208161), JNK (1:1,000; cat. no. ab179461), phosphorylated (p)-JNK (1:2,000; cat. no. ab219584), c-JUN (1:1,000; cat. no. ab40766), p-c-JUN (1:1,000; cat. no. ab227533), Cyt-c (1:1,000; cat. no. ab133504) and GAPDH (1:2,000; cat. no. ab8245) antibodies (all from Abcam) were added to the membrane and incubated at $4^{\circ} \mathrm{C}$ overnight. After washing the membrane, horseradish peroxidase-labeled IgG secondary antibodies (1:5,000; cat. no. A0208; Beyotime Institute of Biotechnology) were added and incubated at $37^{\circ} \mathrm{C}$ for $2 \mathrm{~h}$. After washing the membrane, BeyoECL Moon (Beyotime Institute of Biotechnology) reagent was added, and the protein bands were semi-quantified with LabWorks 4.5 software (Analytik Jena AG). The ratio of target protein band to internal reference band (GAPDH) was calculated.

Statistical analysis. The experimental results are shown as the mean \pm SD, and data were analyzed using SPSS 23.0 software (IBM Corp.). The results of the MWM test were analyzed by mixed ANOVA. The differences in other parameters were analyzed by one-way ANOVA, and Tukey's test was used for post-hoc comparisons. $\mathrm{P}<0.05$ was considered to indicate a statistically significant difference.

\section{Results}

Effects of exenatide on blood glucose, body weight and insulin in DM model rats. After the 16-week experiment, the blood glucose of diabetic rats was significantly higher compared with that in the CON group (Fig. 1A). Compared with that in the DM group, the blood glucose of the Ex + DM group exhibited a downward trend $(\mathrm{P}=0.0013$; Fig. 1A). Blood glucose in the first week was not baseline data; therefore, blood glucose in the control and model groups were not the same. Compared with the body weight of the CON rats, the weight of DM model rats was significantly decreased $(\mathrm{P}=0.0019$; Fig. 1B). Following treatment with exenatide, the body weight of rats in the Ex + DM group was significantly decreased compared with that of rats in the DM group ( $\mathrm{P}=0.0021$; Fig. 1B). Moreover, rats in the $\mathrm{DM}$ group displayed higher insulin levels compared with those in the CON group $(\mathrm{P}=0.0008)$, whereas the insulin levels after exenatide treatment showed a significant decrease $(\mathrm{P}=0.0014$; Fig. 1C). These results suggested that exenatide improved blood glucose regulation and reduced body weight and fasting insulin levels. Moreover, the rats in the DM group presented higher cholesterol ( $\mathrm{P}=0.0102$; Fig. 1D) and triglyceride $(\mathrm{P}=0.0022$; Fig. 1E) levels compared with those of the rats in the CON group; however, the serum levels of cholesterol $(\mathrm{P}=0.0007$; Fig. 1D) and triglyceride $(\mathrm{P}=0.0036$; Fig. 1E) were reduced following exenatide treatment. The results showed 
A
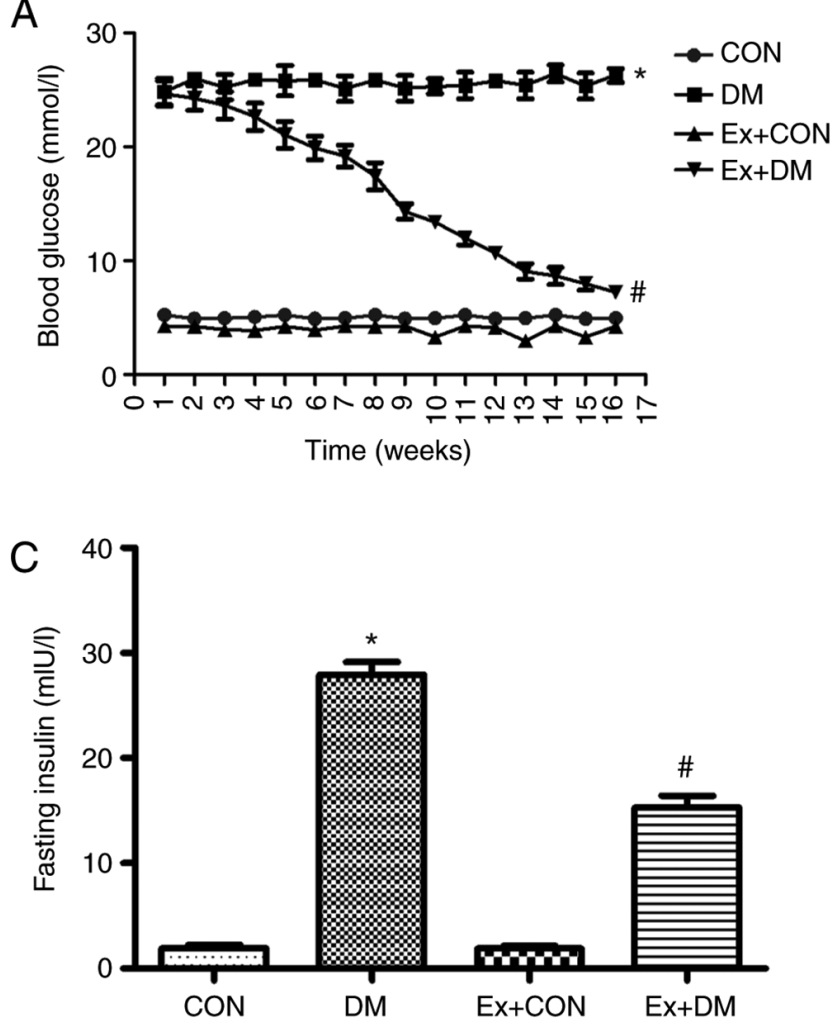

B

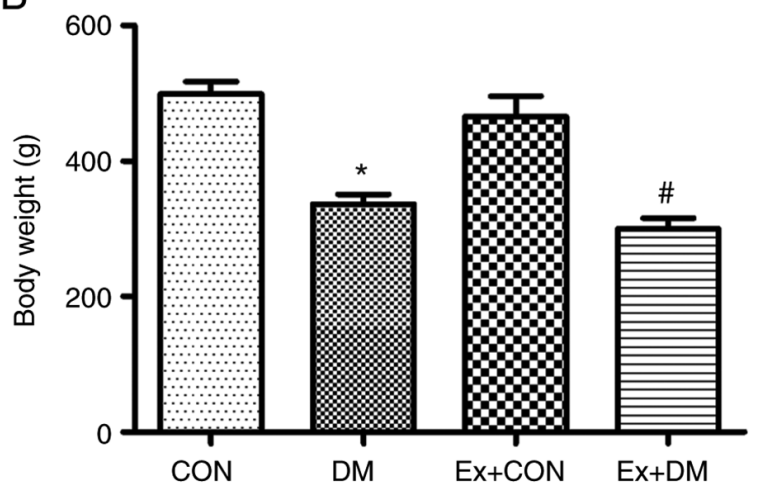

$\mathrm{D}$

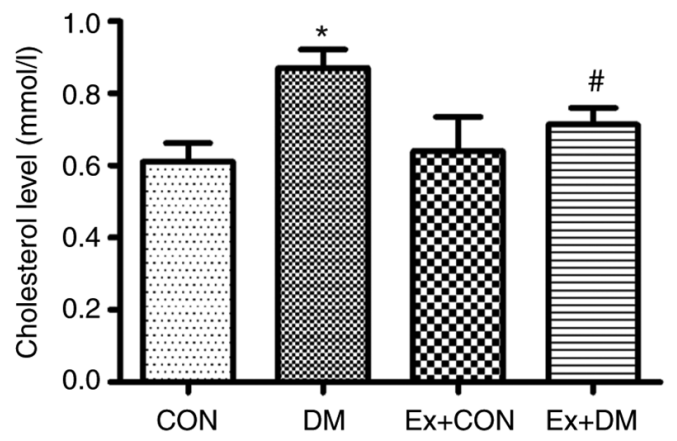

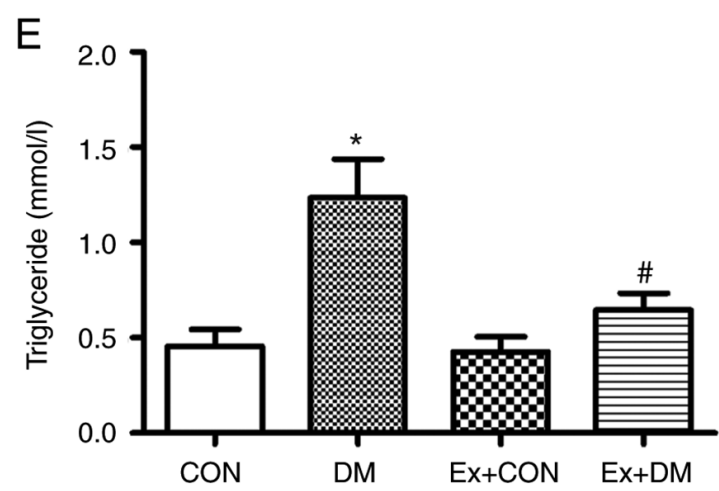

Figure 1. Effect of exenatide on blood biochemical indexes and body weight. (A) Blood glucose levels, (B) body weight, (C) insulin levels, (D) cholesterol levels and $(\mathrm{E})$ triglyceride levels among the groups. Data are presented as the mean $\pm \mathrm{SD} ; \mathrm{n}=15 ;{ }^{*} \mathrm{P}<0.05$ vs. $\mathrm{CON} ;{ }^{\prime \prime} \mathrm{P}<0.05$ vs. DM. CON, control; DM, diabetes mellitus; Ex, exenatide.

that exenatide effectively regulated lipid levels in the body, thereby reducing the incidence of cardiovascular disease.

Effect of exenatide on learning and memory ability in rats with $D M$. To investigate whether exenatide could ameliorate the spatial memory impairment of rats with DM, the MWM hidden platform task was performed to assess hippocampus-dependent cognitive capacity. The effects of exenatide on learning and memory ability during latency trials are shown in Fig. 2A. The DM model rats required more time to find the hidden platform on days 1-5 ( $\mathrm{P}=0.0019, \mathrm{P}=0.0011, \mathrm{P}=0.0010$, $\mathrm{P}=0.0009$ and $\mathrm{P}=0.0007$, respectively); however, the $\mathrm{Ex}+\mathrm{DM}$ rats displayed a significantly shorter latency time than the DM rats $(\mathrm{P}=0.0053, \mathrm{P}=0.0049, \mathrm{P}=0.0051, \mathrm{P}=0.0037$ and $\mathrm{P}=0.0026$, respectively; Fig. 2A). In the probe test, the platform was removed. In DM rats, the time spent in the target quadrant was significantly decreased ( $\mathrm{P}=0.0017$; Fig. $2 \mathrm{~B})$; however, rats in the $\mathrm{Ex}+\mathrm{DM}$ group spent more time in the goal quadrant compared with those in the DM group ( $\mathrm{P}=0.0032$; Fig. 2B). Nevertheless, there was no significant difference in swimming speed among the groups $(\mathrm{P}=0.0875$; Fig. $2 \mathrm{C})$. These findings are consistent with those of a previous study (25) and indicated that exenatide may be suitable for treatment of DM-induced memory deficits.

Effect of exenatide on hippocampal neuron morphology in rats with DM. 10 high magnification fields were selected for each section, the number of positive cells was counted and the average value was calculated. Morphological changes in hippocampal neurons were observed by H\&E staining among the groups. Karyopyknosis and nucleoli disappearance were observed in the hippocampal neurons of rats in the $\mathrm{DM}$ group ( $\mathrm{P}=0.0012$; Fig. $3 \mathrm{~A})$. In the $\mathrm{DM}$ model rats treated with exenatide, the hippocampal neuron structure 

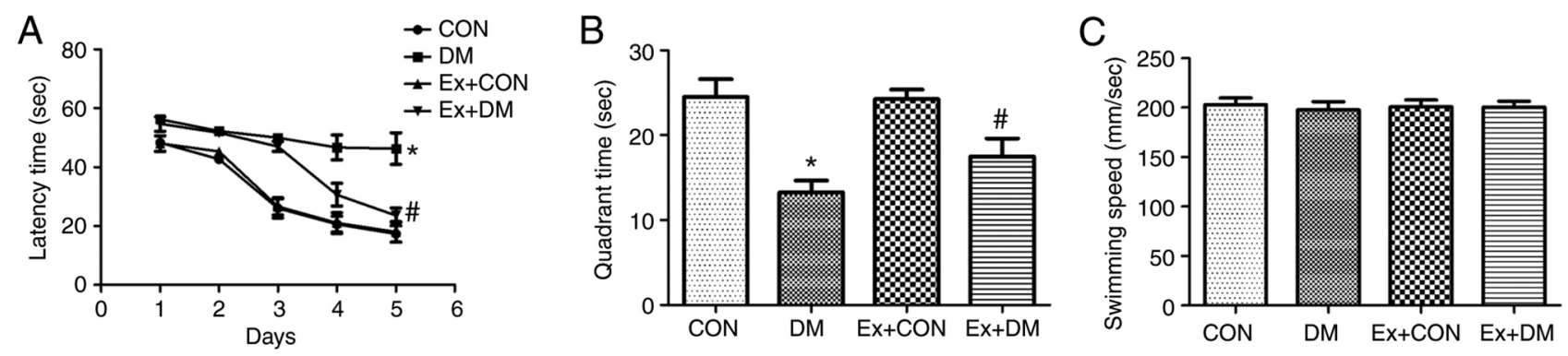

Figure 2. Effect of exenatide on spatial learning and memory. (A) Escape latency of rats in each group. (B) Changes in the duration of time spent in the target quadrant. (C) Swimming speed of rats in each group. Data are presented as the mean $\pm \mathrm{SD} ; \mathrm{n}=15$; ${ }^{*} \mathrm{P}<0.05$ vs. CON; ${ }^{\#} \mathrm{P}<0.05$ vs. DM. CON, control; DM, diabetes mellitus; Ex, exenatide.
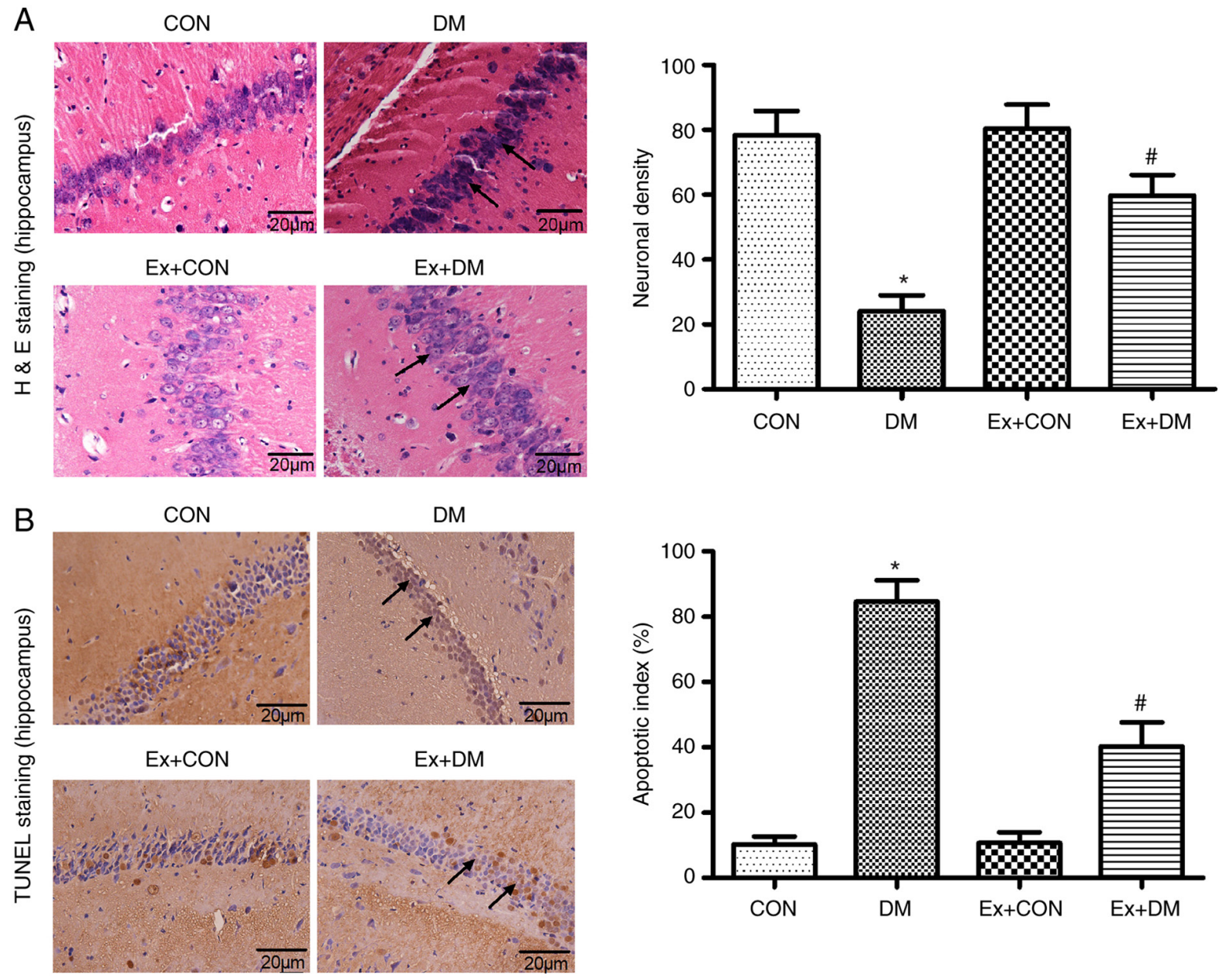

Figure 3. Pathological changes in H\&E-stained and TUNEL-stained hippocampal sections. (A) Representative images of H\&E staining from each group (magnification, $\mathrm{x} 400$ ) and neuronal density in each group calculated from these images. (B) Representative images of TUNEL staining in hippocampal brain slices (magnification, $\mathrm{x} 400$ ) and apoptotic index of rat neurons in each group calculated from these images. Black arrows indicate morphological characteristics and apoptotic cells. Data are presented as the mean $\pm \mathrm{SD} ; \mathrm{n}=5 ;{ }^{*} \mathrm{P}<0.05$ vs. CON; ${ }^{\prime} \mathrm{P}<0.05$ vs. DM. CON, control; DM, diabetes mellitus; Ex, exenatide; $\mathrm{H} \& \mathrm{E}$, hematoxylin and eosin.

was relatively intact, the number of neurons was higher than that in rats in the DM group, and the cytoplasmic shrinkage was recovered (Fig. 3A). These results suggested that exenatide exhibited protective effects on the morphology of hippocampal neurons.

Effect of exenatide on apoptosis of hippocampal neurons. The anti-apoptotic effects of exenatide on diabetic rats was investigated. The ratio of apoptotic cells to total cell number was calculated following TUNEL staining, and the results revealed that there were more apoptotic cells in the DM group compared with in the CON group $(\mathrm{P}=0.0025)$; however, neuronal apoptosis was significantly alleviated after exenatide administration ( $\mathrm{P}=0.0017$; Fig. $3 \mathrm{~B})$. The slight difference between the CON group and the Ex + CON group was not statistically significant $(\mathrm{P}=0.0981)$. 

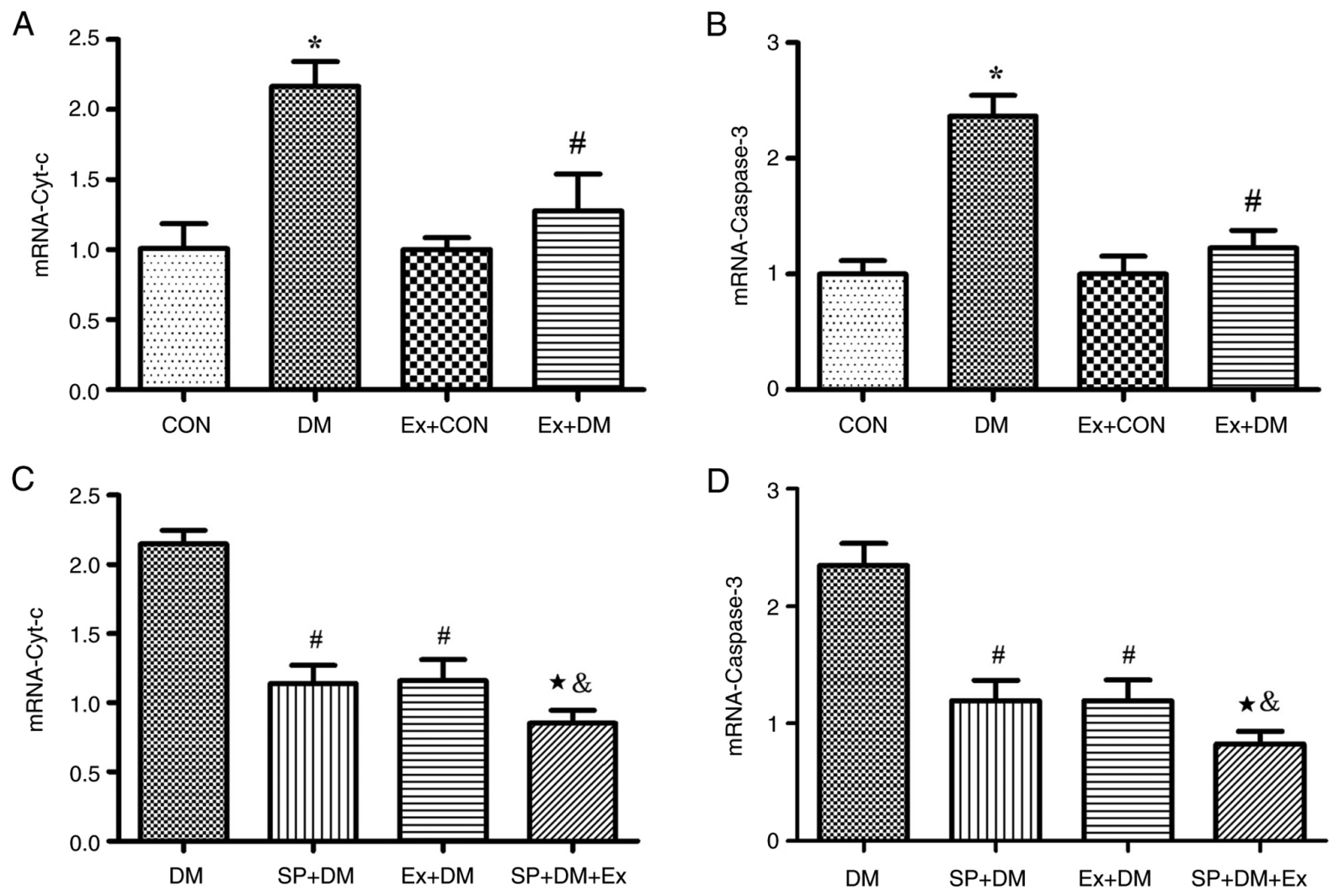

Figure 4. Effect of exenatide on the mRNA expression levels of Caspase-3 and Cyt-c. mRNA expression levels of (A and C) Cyt-c and (B and D) Caspase-3. Data are presented as the mean $\pm \mathrm{SD} ; \mathrm{n}=5 ;{ }^{\mathrm{P}} \mathrm{P}<0.05$ vs. CON; ${ }^{\sharp} \mathrm{P}<0.05$ vs. $\mathrm{DM} ;{ }^{\star} \mathrm{P}<0.05$ vs. the $\mathrm{SP}+\mathrm{DM} ;{ }^{\circledR} \mathrm{P}<0.05$ vs. Ex $+\mathrm{DM}$.

Moreover, the results of RT-qPCR revealed that the mRNA expression levels of Cyt-c ( $\mathrm{P}=0.0011$; Fig. 4A) and Caspase-3 $(\mathrm{P}=0.0018$; Fig. 4B) were higher in the DM group compared with those in the CON group. Exenatide lowered the mRNA expression of Cyt-c and Caspase-3. Furthermore, western blotting was performed to detect the expression levels of the apoptosis-related proteins Caspase-3 and Cyt-c in hippocampal tissues. As shown in Fig. 5A, compared with those in the DM group, the expression levels of Caspase- 3 and Cyt-c were significantly decreased in the Ex + DM group. mRNA $(\mathrm{P}=0.0008, \mathrm{P}=0.0014$; Fig. $4 \mathrm{C}$ and $\mathrm{D})$ and protein $(\mathrm{P}=0.0021$, $\mathrm{P}=0.0013$; Fig. 5B) levels of Cyt-c and Caspase-3 were significantly decreased in diabetic rats treated with SP600125. The results suggested that exenatide could inhibit neuronal apoptosis induced by diabetes.

Effect of exenatide on Caspase-3 activity in diabetic rats. Caspase-3 activity in the DM group was significantly higher compared with that in the CON group $(\mathrm{P}=0.0016$; Fig. 5C). These results indicated that diabetes could lead to enhanced Caspase- 3 activity. By contrast, Caspase- 3 activity was significantly decreased in diabetic rats after exenatide administration ( $\mathrm{P}=0.0020$; Fig. $5 \mathrm{C}$ ), indicating that exenatide could significantly inhibit the diabetes-induced increase in Caspase- 3 activity.

Effects of exenatide on the JNK/c-JUN signaling pathway. The protein expression levels of JNK, p-JNK, c-JUN and p-c-JUN were detected in hippocampal tissues by western blotting. The protein expression levels of p-JNK and p-c-JUN were significantly higher in the DM group compared with those in the $\mathrm{CON}$ group $(\mathrm{P}=0.0048$ and $\mathrm{P}=0.0011$, respectively; Fig. 6A). Conversely, the expression levels of these proteins were significantly reduced in the $\mathrm{Ex}+\mathrm{DM}$ group $(\mathrm{P}=0.0051$ and $\mathrm{P}=0.0043$, respectively; Fig. $6 \mathrm{~A})$. The findings indicated that exenatide may inhibit neuronal apoptosis via the JNK/c-JUN signaling pathway.

To further explore the protective effect of exenatide on the ERS signaling pathway, rats were treated with a specific inhibitor of JNK (SP600125) $30 \mathrm{~min}$ before modeling and the changes in p-JNK and p-c-JUN expression were detected. Compared with those in the DM group, the protein expression levels of p-JNK and p-c-JUN were significantly decreased in the $\mathrm{SP}+\mathrm{DM}$ group $(\mathrm{P}=0.0023$ and $\mathrm{P}=0.0082$, respectively; Fig. 6B). Notably, p-JNK and p-c-JUN were expressed at similar levels in the Ex+DM and SP+DM groups, suggesting that exenatide and SP600125 have similar inhibitory effects on the JNK pathway. This conclusion was further verified in the $\mathrm{SP}+\mathrm{DM}+\mathrm{Ex}$ group, as p-JNK and p-c-JUN were expressed at half the level of the Ex+DM and SP+DM group. These results indicated that exenatide may reduce neuronal apoptosis in rats with diabetes by inhibiting JNK/c-JUN signaling.

\section{Discussion}

Diabetic encephalopathy is characterized by acquired cognitive and behavioral deficits, and includes pathological, morphological, electrophysiological, neurobiochemical, neuropsychological and behavioral changes. Studies have shown that diabetes can cause MCI, which is a state between 

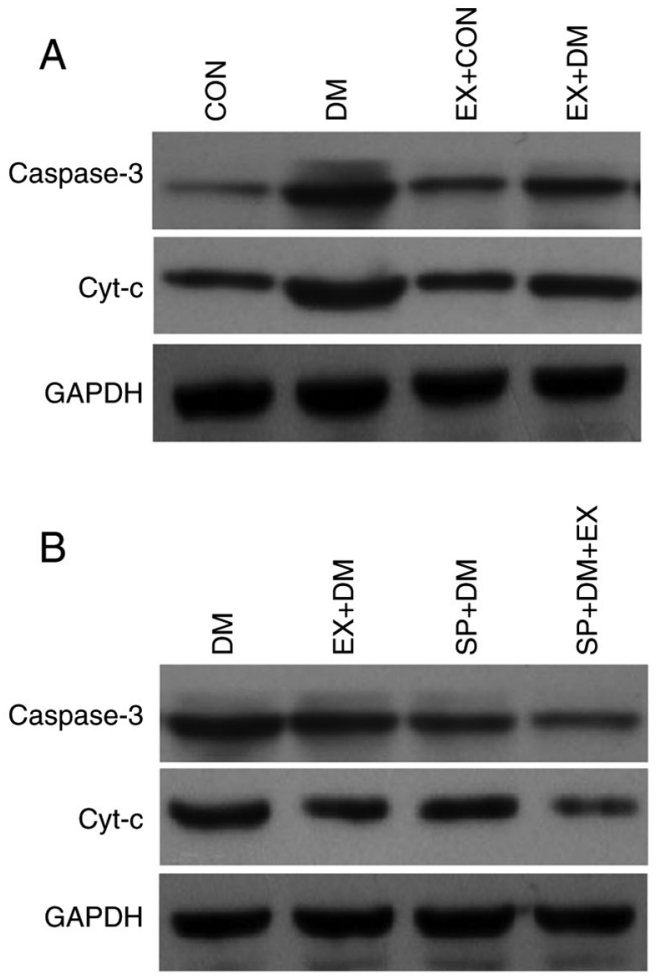
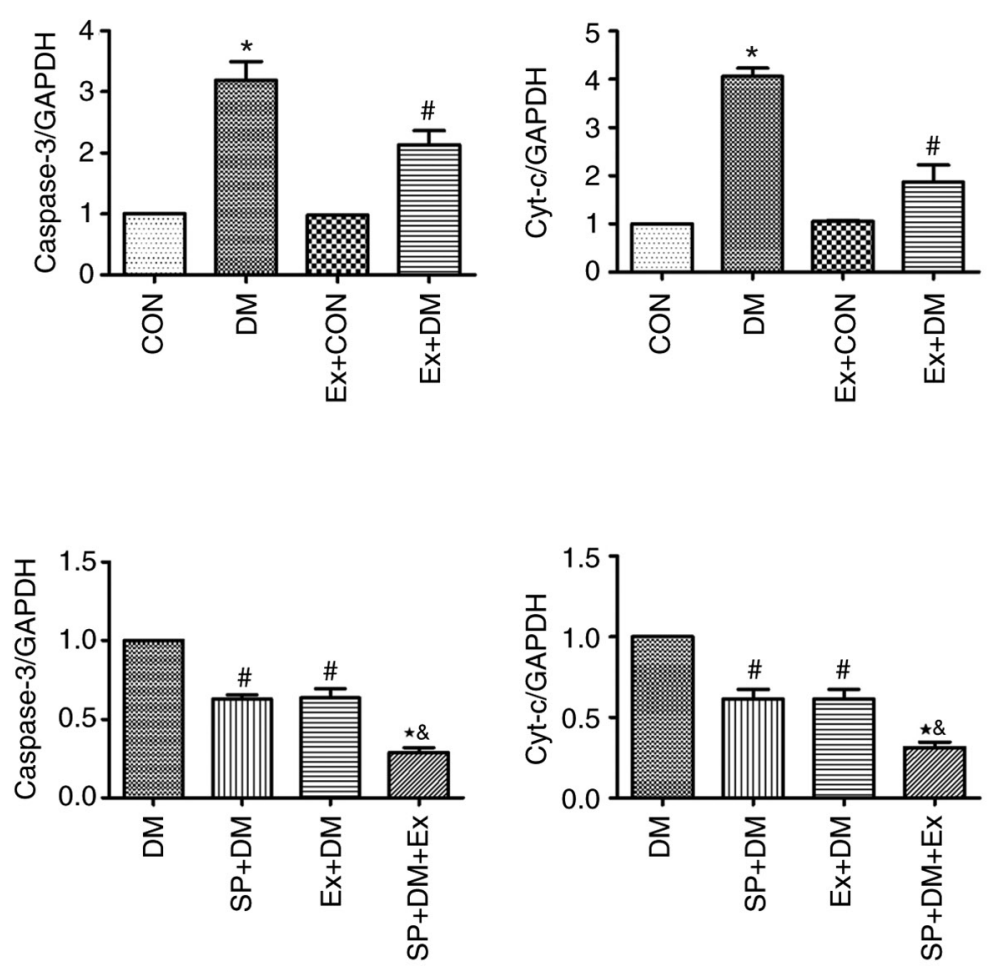

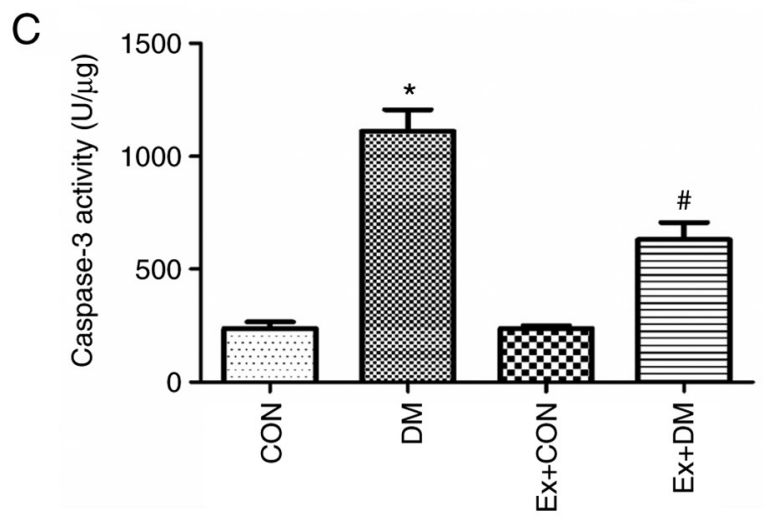

Figure 5. Effects of exenatide on Caspase-3 and Cyt-c protein expression levels and Caspase-3 activity. Representative western blots and semi-quantification of the protein expression levels of Caspase-3 and Cyt-c (A) in response to exenatide and (B) in response to exenatide and SP600125. (C) Activity of Caspase-3 in the brain tissue of rats in each group. Data are presented as the mean $\pm \mathrm{SD} ; \mathrm{n}=5 ;{ }^{*} \mathrm{P}<0.05$ vs. $\mathrm{CON} ;{ }^{\sharp} \mathrm{P}<0.05 \mathrm{vs}$. DM; ${ }^{\star} \mathrm{P}<0.05$ vs. $\mathrm{SP}+\mathrm{DM} ;{ }^{\circledR} \mathrm{P}<0.05$ vs. Ex $+\mathrm{DM}$.

normal cognitive function and dementia $(26,27)$. The earliest and largest cross-sectional study of the association between diabetes and dementia revealed that the relative risk values of diabetes with Alzheimer's disease and vascular dementia were as high as 1.3-2.2 and 2.0-3.4, respectively $(28,29)$.

In patients with diabetes, insufficient insulin secretion and insulin resistance can lead to hyperglycemia. Increased blood sugar levels can accelerate the onset of Alzheimer's disease, with diabetes being closely associated with Alzheimer's disease (30). Epidemiological studies have revealed that elderly individuals with diabetes have twice the risk of developing cognitive dysfunction and other dementia symptoms compared with the risk among the general population, and diabetes is closely associated with Alzheimer's disease $(31,32)$. Cognitive impairment associated with diabetes causes a great inconvenience for patients; therefore, it is of great clinical significance to carry out research with the aim of preventing cognitive impairment in diabetes. In the treatment of type 2 diabetes, exenatide is effective in controlling blood glucose, improving the function of pancreatic $\beta$ cells, increasing insulin sensitivity, reducing body mass in patients with obesity, and regulating inflammatory mediators and lipids, thereby reducing the incidence of cardiovascular disease, hypoglycemia and pancreatitis (33). In a rat model of Alzheimer's disease, GLP-1 reduced endogenous $\beta$-amyloid levels and inhibited the A $\beta$-induced apoptosis of nerve cells (34). GLP-1 and its long-acting counterpart, exendin- 4 , have also been reported to inhibit glutamate-induced apoptosis of hippocampal neurons; however, the specific mechanism by which GLP-1 interferes with neuronal apoptosis remains unclear (35).

JNK is a member of the MAPK family and serves an important role in cell apoptosis, growth, differentiation, 

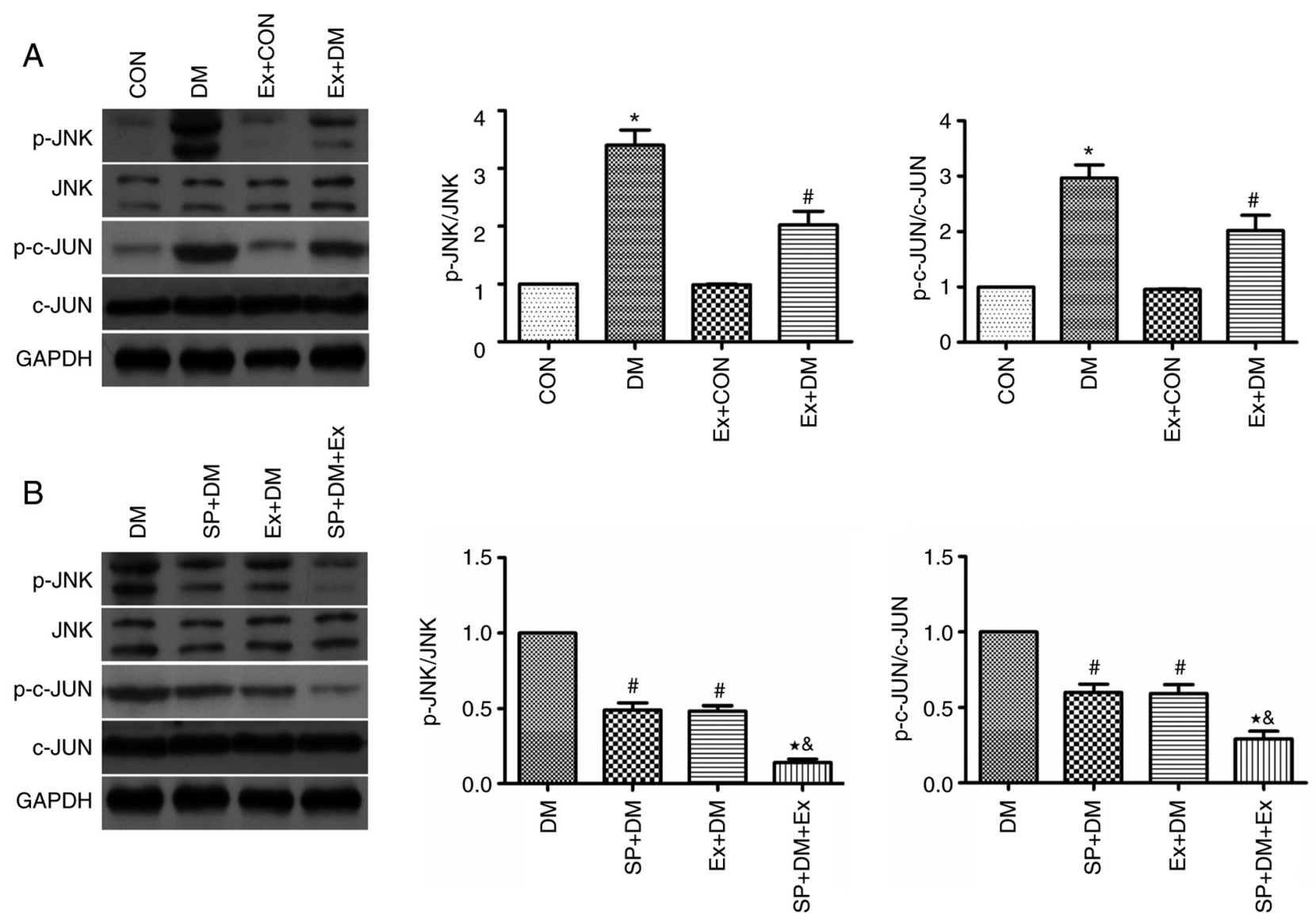

Figure 6. Effect of exenatide on the expression levels of JNK, p-JNK, c-JUN and p-c-JUN. Representative western blot and semi-quantification of the protein expression levels of p-JNK, JNK, p-c-JUN and c-JUN (A) in response to exenatide and (B) in response to exenatide and SP600125 (a specific inhibitor of JNK). Data are presented as the mean $\pm \mathrm{SD} ; \mathrm{n}=5 ;{ }^{*} \mathrm{P}<0.05$ vs. CON; ${ }^{\sharp} \mathrm{P}<0.05$ vs. $\mathrm{DM} ;{ }^{\star} \mathrm{P}<0.05$ vs. SP + DM; ${ }^{\circledR} \mathrm{P}<0.05$ vs. Ex + DM.

embryonic development and immune responses. The JNK signaling pathway can be activated by various cellular stimuli, such as ultraviolet radiation, heat shock or inflammatory stimuli, through the MAPKKK-MAPKK-MAPK (JNK) cascade system $(36,37)$. c-JUN is a member of the HEG family of fast-reacting genes and can serve a role in the activated protein-1 (AP-1) complex to regulate cell proliferation, differentiation and apoptosis (38). Under the action of JNK, Ser63 and Ser73 in the N-terminal active region of JUN are phosphorylated, self-translocation occurs, and the activity is upregulated to promote the activity of AP-1; activated JUN further promotes the expression of a variety of pro-apoptotic proteins (39). The results of the present study revealed that, in STZ-induced diabetic rats with learning and memory impairment, JNK and c-JUN proteins in the hippocampus were phosphorylated, and exenatide could reduce the expression levels of p-JNK and p-c-JUN, thus suggesting that the high glucose environment in the body may activate the JNK/c-JUN signaling pathway.

The metabolic dysfunction caused by the imbalance of the endocrine system and the activation of a large number of inflammatory mediators under stress can lead to the opening of the mitochondrial permeability pores to release Cyt-c and activate Caspase-3 to initiate the apoptosis program (40). Caspase-3 is an apoptotic factor that can be activated by an upstream initiator. After activation, Caspase-3 forms an apoptotic protein that acts on specific substrates to cause morphological and biochemical changes in cells, leading to apoptosis. Regulating blood sugar, inhibiting the opening of mitochondrial permeability transition pores, and reducing the release of Cyt-c, thereby blocking the apoptotic pathway at the level of Caspase-3, can effectively protect hippocampal neurons (41). The results of the present study revealed that the expression levels of Cyt-c and Caspase-3 in the hippocampus of rats with diabetic cognitive dysfunction were significantly increased, suggesting that the high-glycemic environment in the body may upregulate Cyt-c and Caspase-3 expression levels, thereby inducing hippocampal neuron apoptosis. By contrast, exenatide could inhibit the process and reduce the expression levels of Cyt-c and Caspase-3. Currently, exenatide is used to regulate blood sugar in patients with type 2 diabetes, improve insulin sensitivity and effectively control body weight; however, the application of exenatide to reverse cognitive impairment in diabetes is not very common. Therefore, more experiments are required to confirm the effectiveness and safety of exenatide for cognitive impairment. Moreover, exenatide treatment of type 2 diabetes often results in adverse gastrointestinal reactions, such as nausea, loss of appetite and vomiting (42). Therefore, further investigation is required to improve the safety of exenatide therapy in clinical practice.

In conclusion, in the process of cognitive dysfunction caused by diabetes, along with activation of the JNK/c-JUN signaling pathway, the expression levels of Cyt-c and Caspase-3 are significantly increased. Therefore, it was hypothesized that 
regulation of the JNK/c-JUN signaling pathway may lead to the upregulation of the expression of apoptotic proteins, which may be one of the factors involved in hippocampal neuronal apoptosis leading to diabetic cognitive impairment. Notably, exenatide inhibited the phosphorylation of JNK and c-JUN, and downregulated the expression levels of Cyt-c and Caspase- 3 . These findings indicated that the suppression of apoptosis-induced diabetic cognitive impairment by exenatide may be achieved through its inhibition of the JNK/c-JUN signaling pathway.

\section{Acknowledgements}

Not applicable.

\section{Funding}

The experiment was funded by Hebei Provincial Health Commission (grant no. 20200130) and the Startup Foundation for Advanced Talents (grant no. 2019GC18).

\section{Availability of data and materials}

The datasets used and/or analyzed during the current study are available from the corresponding author on reasonable request.

\section{Authors' contributions}

GW and MY confirm the authenticity of all the raw data. GW and MY designed the experiments. ZZ and BR made substantial contributions to acquisition of data. JL and WY made contributions to the analysis of data. DS, LW and XZ performed the experiments. All authors read and approved the final version of the manuscript.

\section{Ethics approval and consent to participate}

The present study was approved by the Ethics Committee of North China University of Science and Technology (approval no. LAEC-NCST-20200002).

\section{Patient consent for publication}

Not applicable.

\section{Competing interests}

The authors declare that there are no competing interests.

\section{References}

1. Prieto-Gómez B, Díaz-Vázquez $\mathrm{M}$ and Pérez-Torres D: Hippocampal electrophysiological changes during the elicited metabolic syndrome in Wistar rats. Metabol Open 5: 100027, 2020.

2. Meng Y, Wang W, Kang J, Wang X and Sun L: Role of the $\mathrm{PI} 3 \mathrm{~K} / \mathrm{AKT}$ signalling pathway in apoptotic cell death in the cerebral cortex of streptozotocin-induced diabetic rats. Exp Ther Med 13: 2417-2422, 2017.

3. Moran C, Beare R, Wang W, Callisaya M and Srikanth V; Alzheimer's Disease Neuroimaging Initiative (ADNI): Type 2 diabetes mellitus, brain atrophy, and cognitive decline. Neurology 92: e823-e830, 2019.
4. Chornenkyy Y, Wang WX, Wei A and Nelson PT: Alzheimer's disease and type 2 diabetes mellitus are distinct diseases with potential overlapping metabolic dysfunction upstream of observed cognitive decline. Brain Pathol 29: 3-17, 2019.

5. Hassing LB, Grant MD, Hofer SM, Pedersen NL, Nilsson SE, Berg S, McClearn G and Johansson B: Type 2 diabetes mellitus contributes to cognitive decline in old age: A longitudinal population-based study. J Int Neuropsychol Soc 10: 599-607, 2004.

6. Mayeda ER, Haan MN, Yaffe K, Kanaya AM and Neuhaus J: Does type 2 diabetes increase rate of cognitive decline in older Mexican Americans? Alzheimer Dis Assoc Disord 29: 206-212, 2015.

7. Yanagawa $M$, Umegaki $H$, Makino $T$, Nakashima $H$ and Kuzuya M: Neuropsychological differences in Alzheimer's disease patients with or without type 2 diabetes mellitus. Geriatr Gerontol Int 16: 1232-1235, 2016.

8. Blalock EM, Phelps JT, Pancani T, Searcy JL, Anderson KL, Gant JC, Popovic J, Avdiushko MG, Cohen DA, Chen KC, et al: Effects of long-term pioglitazone treatment on peripheral and central markers of aging. PLoS One 5: e10405, 2010.

9. Duarte JM: Metabolic alterations associated to brain dysfunction in diabetes. Aging Dis 6: 304-321, 2015.

10. Calvo-Ochoa E and Arias C: Cellular and metabolic alterations in the hippocampus caused by insulin signalling dysfunction and its association with cognitive impairment during aging and Alzheimer's disease: Studies in animal models. Diabetes Metab Res Rev 31: 1-13, 2015.

11. Ma P, Mao XY, Li XL, Ma Y, Qiao YD, Liu ZQ, Zhou HH and Cao YG: Baicalin alleviates diabetes-associated cognitive deficits via modulation of mitogen-activated protein kinase signaling, brain-derived neurotrophic factor and apoptosis. Mol Med Rep 12: 6377-6383, 2015.

12. Sowndhararajan K, Deepa P, Kim M, Park SJ and Kim S: Neuroprotective and cognitive enhancement potentials of baicalin: A review. Brain Sci 8: 104, 2018.

13. Kaneko M: Molecular pharmacological studies on the protection mechanism against endoplasmic reticulum stress-induced neurodegenerative disease. Yakugaku Zasshi 132: 1437-1442, 2012 (In Japanese).

14. Nomura Y: Pharmacological studies on neurodegenerative diseases focusing on refolding and degradation of unfolded proteins in the endoplasmic reticulum. Yakugaku Zasshi 134: 537-543, 2014 (In Japanese).

15. Deacon CF, Mannucci E and Ahrén B: Glycaemic efficacy of glucagon-like peptide-1 receptor agonists and dipeptidyl peptidase- 4 inhibitors as add-on therapy to metformin in subjects with type 2 diabetes-a review and meta analysis. Diabetes Obes Metab 14: 762-767, 2012.

16. Calanna S, Christensen M, Holst JJ, Laferrère B, Gluud LL, Vilsbøll T and Knop FK: Secretion of glucose-dependent insulinotropic polypeptide in patients with type 2 diabetes: Systematic review and meta-analysis of clinical studies. Diabetes Care 36: 3346-3352, 2013.

17. Chen WJY, Diamant M, de Boer K, Harms HJ, Robbers LFHJ, van Rossum AC, Kramer MHH, Lammertsma A A and Knaapen P: Effects of exenatide on cardiac function, perfusion, and energetics in type 2 diabetic patients with cardiomyopathy: A randomized controlled trial against insulin glargine. Cardiovasc Diabetol 16: 67, 2017.

18. Ravassa S, Zudaire A and Díez J: GLP-1 and cardioprotection: from bench to bedside. Cardiovasc Res 94: 316-323, 2012.

19. Wang XH, Li L, Hölscher C, Pan YF, Chen XR and Qi JS: Val8-glucagon-like peptide-1 protects against A $\beta 1-40$-induced impairment of hippocampal late-phase long-term potentiation and spatial learning in rats. Neuroscience 170: 1239-1248, 2010.

20. Himeno T, Kamiya H, Naruse K, Harada N, Ozaki N, Seino Y, Shibata T, Kondo M, Kato J, Okawa T, et al: Beneficial effects of exendin-4 on experimental polyneuropathy in diabetic mice. Diabetes 60: 2397-2406, 2011.

21. Linnemann AK, Neuman JC, Battiola TJ, Wisinski JA, Kimple ME and Davis DB: Glucagon-like peptide-1 regulates cholecystokinin production in $\beta$-cells to protect from apoptosis. Mol Endocrinol 29: 978-987, 2015.

22. Jin J, Kang HM, Jung J, Jeong JW and Park C: Related expressional change of HIF-1 $\alpha$ to the neuroprotective activity of exendin-4 in transient global ischemia. Neuroreport 25: 65-70, 2014.

23. Islam MS and Loots du T: Experimental rodent models of type 2 diabetes: A review. Methods Find Exp Clin Pharmacol 31: 249-261, 2009 . 
24. Livak KJ and Schmittgen TD: Analysis of relative gene expression data using real-time quantitative PCR and the 2(-Delta Delta C(T)) method. Methods 25: 402-408, 2001.

25. Chen S, Liu AR, An FM, Yao WB and Gao XD: Amelioration of neurodegenerative changes in cellular and rat models of diabetes-related Alzheimer's disease by exendin-4. Age (Dordr) 34: 1211-1224, 2012.

26. Dong Y, Kua ZJ, Khoo EY, Koo EH and Merchant RA: The utility of brief cognitive tests for patients with type 2 diabetes mellitus: A systematic review. J Am Med Dir Assoc 17: 889-895, 2016.

27. Sadanand S, Balachandar R and Bharath S: Memory and executive functions in persons with type 2 diabetes: A meta-analysis. Diabetes Metab Res Rev 32: 132-142, 2016.

28. Dar TA, Sheikh IA, Ganie SA, Ali R, Singh LR, Gan SH, Kamal MA and Zargar MA: Molecular linkages between diabetes and Alzheimer's disease: Current scenario and future prospects. CNS Neurol Disord Drug Targets 13: 290-298, 2014.

29. Kubis-Kubiak AM, Rorbach-Dolata A and Piwowar A: Crucial players in Alzheimer's disease and diabetes mellitus: Friends or foes? Mech Ageing Dev 181: 7-21, 2019.

30. Wang SB and Jia JP: Oxymatrine attenuates diabetes-associated cognitive deficits in rats. Acta Pharmacol Sin 35: 331-338, 2014.

31. Liu J, Feng L, Ma D, Zhang M, Gu J, Wang S, Fu Q, Song Y, Lan Z, $\mathrm{Qu}$ R and Ma S: Neuroprotective effect of paeonol on cognition deficits of diabetic encephalopathy in streptozotocin-induced diabetic rat. Neurosci Lett 549: 63-68, 2013.

32. Liu YW, Zhu X, Lu Q, Wang JY, Li W, Wei YQ and Yin XX: Total saponins from Rhizoma Anemarrhenae ameliorate diabetesassociated cognitive decline in rats: Involvement of amyloid-beta decrease in brain. J Ethnopharmaco 139: 194-200, 2012.

33. DeFronzo RA, Triplitt C, Qu Y, Lewis MS, Maggs D and Glass LC: Effects of exenatide plus rosiglitazone on beta-cel function and insulin sensitivity in subjects with type 2 diabetes on metformin. Diabetes Care 33: 951-957, 2010.

34. Wang ZJ, Han YF, Zhao F, Yang GZ, Yuan L, Cai HY, Yang JT, Holscher C, Qi JS and Wu MN: A dual GLP-1 and Gcg receptor agonist rescues spatial memory and synaptic plasticity in APP/PS1 transgenic mice. Horm Behav 118: 104640, 2020.
35. Perry $\mathrm{T}$ and Greig NH: Enhancing central nervous system endogenous GLP-1 receptor pathways for intervention in Alzheimer's disease. Curr Alzheimer Res 2: 377-385, 2005

36. Lee HN, Shin SA, Choo GS, Kim HJ, Park YS, Kim BS, Kim SK, Cho SD, Nam JS, Choi CS, et al: Anti-inflammatory effect of quercetin and galangin in LPS-stimulated RAW264.7 macrophages and DNCB-induced atopic dermatitis animal models. Int J Mol Med 41: 888-898, 2018

37. Harrison JC, Zyla TR, Bardes ES and Lew DJ: Stress-specific activation mechanisms for the 'cell integrity' MAPK pathway. J Biol Chem 279: 2616-2622, 2004.

38. Huang HM and Liu JC: c-Jun blocks cell differentiation but not growth inhibition or apoptosis of chronic myelogenous leukemia cells induced by STI571 and by histone deacetylase inhibitors. J Cell Physiol 218: 568-574, 2009.

39. Li L, Feng Z and Porter AG: JNK-dependent phosphorylation of c-Jun on serine 63 mediates nitric oxide-induced apoptosis of neuroblastoma cells. J Biol Chem 279: 4058-4065, 2004.

40. Jorda A, Aldasoro M, Aldasoro C, Guerra-Ojeda S, Iradi A, Vila JM, Campos-Campos J and Valles SL: Action of low doses of Aspirin in Inflammation and Oxidative stress induced by a $\beta_{1-42}$ on astrocytes in primary culture. Int J Med Sci 17: 834-843, 2020.

41. Cheng FR, Cui HX, Fang JL, Yuan K and Guo Y: Ameliorative effect and mechanism of the purified anthraquinone-glycoside preparation from rheum palmatum L. on type 2 diabetes mellitus. Molecules 24: 1454, 2019.

42. Vilsbøll T, Christensen M, Junker AE, Knop FK and Gluud LL: Effects of glucagon-like peptide-1 receptor agonists on weight loss: Systematic review and meta-analyses of randomised controlled trials. BMJ 344: d7771, 2012.

This work is licensed under a Creative Commons Attribution-NonCommercial-NoDerivatives 4.0 International (CC BY-NC-ND 4.0) License. 